ARTICLES

nature medicine

\title{
Enhanced T-cell immunogenicity of plasmid DNA vaccines boosted by recombinant modified vaccinia virus Ankara in humans
}

\author{
Samuel J McConkey ${ }^{1,7}$, William H H Reece ${ }^{1,7}$, Vasee S Moorthy ${ }^{1,7}$, Daniel Webster ${ }^{1}$, Susanna Dunachie ${ }^{1}$, \\ Geoff Butcher $^{2}$, Jenni M Vuola ${ }^{1}$, Tom J Blanchard ${ }^{3}$, Philip Gothard ${ }^{1}$, Kate Watkins ${ }^{1}$, Carolyn M Hannan ${ }^{1}$, \\ Simone Everaere ${ }^{1}$, Karen Brown ${ }^{1}$, Kent E Kester ${ }^{4}$, James Cummings ${ }^{4}$, Jackie Williams ${ }^{4}$, D Gray Heppner ${ }^{4}$, \\ Ansar Pathan ${ }^{1}$, Katie Flanagan ${ }^{1}$, Nirmalan Arulanantham ${ }^{1}$, Mark T M Roberts ${ }^{1}$, Michael Roy ${ }^{5}$, Geoffrey L Smith ${ }^{2}$, \\ Joerg Schneider ${ }^{6}$, Tim Peto ${ }^{1}$, Robert E Sinden ${ }^{2}$, Sarah C Gilbert ${ }^{1} \&$ Adrian V S Hill $^{1}$ \\ In animals, effective immune responses against malignancies and against several infectious pathogens, including \\ malaria, are mediated by T cells. Here we show that a heterologous prime-boost vaccination regime of DNA either \\ intramuscularly or epidermally, followed by intradermal recombinant modified vaccinia virus Ankara (MVA), induces \\ high frequencies of interferon (IFN)- $\gamma$-secreting, antigen-specific T-cell responses in humans to a pre-erythrocytic \\ malaria antigen, thrombospondin-related adhesion protein (TRAP). These responses are five- to tenfold higher than \\ the T-cell responses induced by the DNA vaccine or recombinant MVA vaccine alone, and produce partial protection \\ manifest as delayed parasitemia after sporozoite challenge with a different strain of Plasmodium falciparum. Such \\ heterologous prime-boost immunization approaches may provide a basis for preventative and therapeutic \\ vaccination in humans.
}

T cells are central to acquired immunity. They act as effector cells in their own right by secreting cytokines, effecting cytolysis and other mechanisms ${ }^{1,2}$. They can also help B cells generate effective humoral immunity and, in many cases, are needed for this purpose ${ }^{3}$. Until recently, however, most vaccine development efforts were aimed at stimulating B cells and plasma cells to optimize protective antibody responses.

There are several examples of depletion and adoptive transfer experiments in animal models, in which T cells are the critical effector cells. In mouse malaria, IFN- $\gamma$-producing $\mathrm{CD}^{+} \mathrm{T}$ cells have a key role ${ }^{4}$, and clearance of hepatitis B virus (HBV) in animal models also requires $\mathrm{CD}^{+}{ }^{+} \mathrm{T}$ cells $\mathrm{s}^{5}$. Simian models of human immunodeficiency virus infection indicate that $\mathrm{CD}^{+}$and $\mathrm{CD} 4^{+} \mathrm{T}$ lymphocytes are important for control of viremia ${ }^{6}$. The regression of tumors occurs after transfer of $\mathrm{T}$ cells, in several models such as melanoma ${ }^{7}$, colon cancer ${ }^{8}$, renal cell carcinoma $^{9}$ and human papilloma virus-16-induced cervical cancer ${ }^{10}$. Observations in humans also confirm the central role of $\mathrm{T}$ cells. Clearance of HBV in humans, either spontaneously or after lamivudine or interferon treatment, is associated with $\mathrm{HBV}$-specific $\mathrm{CD}^{+} \mathrm{T}$ cells in peripheral blood ${ }^{11,12}$. DNA vaccines used alone induce $\mathrm{T}$-cell responses in animals, as can antigen with many adjuvants. These strategies have not proved as immunogenic in humans as in nonhuman primates or rodents ${ }^{13,14}$. Various strategies have been considered to improve DNA vaccines, such as cytokine augmentation and ballistic epidermal delivery $^{15}$, but induced T-cell responses with DNA vaccines, viral vectors ${ }^{16}$ and protein adjuvant formulations in humans remain modest ${ }^{17}$.

We have previously shown induction of strong protective T-cell responses to subunit vaccines in animals using a mouse model of malaria with a heterologous prime-boost immunization regime ${ }^{18}$. These observations have been extended to other animal models and other diseases ${ }^{19-24}$. Vaccines that produce high T-cell responses can control infection by simian immunodeficiency virus, which is a close relative of human immunodeficiency virus ${ }^{25}$. The work presented here represents the first study of this approach in humans.

Malaria is an increasingly uncontrolled public health problem; $1-3$ million people die annually from Plasmodium falciparum infection ${ }^{26}$. A preventative vaccine is likely to be among the most effective means for its control. Two lines of evidence implicate $\mathrm{T}$ lymphocytes in the immunological control of pre-erythrocytic malaria infection in humans. Severe malaria is less likely in West African children expressing HLA-B ${ }^{\star} 53$ (ref. 27), suggesting a role for HLA class I-restricted T cells in protective immunity. Human irradiated sporozoite-induced immunity is associated with cellular responses ${ }^{28}$.

${ }^{1}$ Nuffield Department of Medicine, University of Oxford, John Radcliffe Hospital, Oxford, OX3 9DU, UK. ${ }^{2}$ Biological Sciences Department, Imperial College, London SW7 2AZ, UK. ${ }^{3}$ Sir William Dunn School of Pathology, University of Oxford, South Parks Road, Oxford OX1 3RE, UK. ${ }^{4}$ Malaria Vaccine Program, Walter Reed Army Institute of Research, 503 Robert Grant Avenue, Silver Spring, Maryland 20910, USA. ${ }^{5}$ PowderJect Vaccines, 585 Science Drive Suite C, Madison, Wisconsin 53711, USA. ${ }^{6}$ Oxxon Pharmaccines, Oxford BioBusiness Centre, Oxford, OX4 4SS, UK. ${ }^{7}$ These authors contributed equally to this work. Correspondence should be addressed to A.V.S.H. (adrian.hill@imm.ox.ac.uk). 


\section{ARTICLES}

a

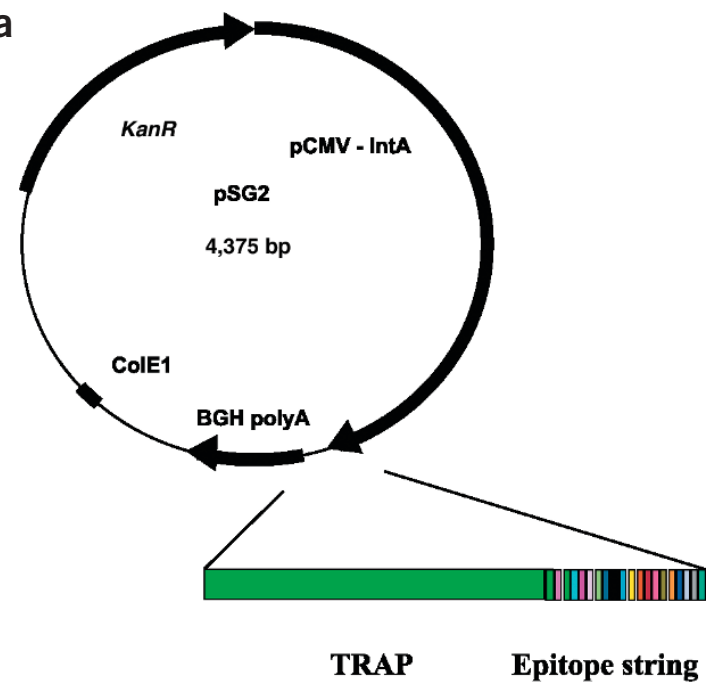

Figure 1 Plasmid DNA vaccine encoding ME-TRAP (a) Schematic representation of the contents of plasmid DNA vaccine used. The same insert was expressed by the MVA vaccine. (b) Composition of the insert in the DNA and MVA vaccines. The epitope string, but not TRAP, was codonoptimized for mammalian translation.

In contrast to most traditional vaccination strategies, which are directed toward the humoral arm of the immune system, vaccine development efforts for pre-erythrocytic stages of malaria have been mainly directed toward inducing cellular immunity, based largely on findings in animal models ${ }^{29}$. We have designed vaccines against the pre-erythrocytic stages of $P$. falciparum, using as vectors plasmid DNA and recombinant MVA. Here we present the results of their evaluation in a series of sequential small clinical trials showing that they are well tolerated, highly immunogenic for $\mathrm{T}$ cells and partly effective in controlling malaria in a high-dose human challenge model using a parasite strain different from the challenge strain. b

\begin{tabular}{|c|c|c|c|c|}
\hline $\begin{array}{l}\text { Epitope/ } \\
\text { protein }\end{array}$ & Amino acid sequence & Antigen & Type & $\begin{array}{l}\text { HLA } \\
\text { restriction }\end{array}$ \\
\hline Is8 & KPNDKSLY & LSA1 & CD8 & $B * 35$ \\
\hline cp26 & KPKDELDY & CSP & $\mathrm{CD} 8$ & $B * 35$ \\
\hline Is6 & KPIVQYDNF & LSA1 & $\mathrm{CD} 8$ & $B * 53$ \\
\hline $\operatorname{tr} 42 / 43$ & ASKNKEKALII & TRAP & CD8 & $B * 8$ \\
\hline $\operatorname{tr} 39$ & GIAGGLALL & TRAP & CD8 & $A^{*} 0201$ \\
\hline cp6 & MNPNDPNRNV & CSP & CD8 & $B * 7$ \\
\hline st8 & MINAYLDKL & STARP & CD8 & $A^{*} 0202$ \\
\hline Is50 & ISKYEDEI & LSA1 & $\mathrm{CD} 8$ & $B * 17$ \\
\hline $\operatorname{tr} 26$ & HLGNVKYLV & TRAP & $\mathrm{CD} 8$ & $A^{*} 0201$ \\
\hline Is53 & KSLYDEHI & LSA1 & CD8 & $B * 58$ \\
\hline $\operatorname{tr} 29$ & LLMDCSGSI & TRAP & $\mathrm{CD} 8$ & $A^{*} 0202$ \\
\hline cp39 & YLNKIQNSL & $\mathrm{CSP}$ & $\mathrm{CD} 8$ & $A^{*} 0201$ \\
\hline la72 & MEKLKELEK & LSA3 & $\mathrm{CD} 8$ & $B * 8$ \\
\hline ex23 & ATSVLAGL & Exp1 & CD8 & $B * 58$ \\
\hline CSP & $\begin{array}{l}\text { DPNANPNVD- } \\
\text { PNANPNV }\end{array}$ & CSP & CD4 & Multiple \\
\hline TRAP AM & $\begin{array}{l}\text { DEWSPCSVTC- } \\
\text { GKGTRSRKRE }\end{array}$ & TRAP & $\begin{array}{l}\text { Heparin- } \\
\text { binding } \\
\text { motif }\end{array}$ & Multiple \\
\hline$(N A N P)_{n}$ & $\begin{array}{l}\text { NANPNANPNA- } \\
\text { NPNANP }\end{array}$ & CSP & B cell & Multiple \\
\hline $38 \mathrm{H} \mathrm{BCG}$ & QVHFQPLPPAVVKL & $B C G$ & CD4 & Multiple \\
\hline FTTp & QFIKANSKFIGITE & $\mathrm{TT}$ & $\mathrm{CD} 4$ & Multiple \\
\hline Pb9 & SYIPSAEKI & $P b C S$ & CD8 & Mouse $\mathrm{H} 2-\mathrm{K}^{\mathrm{d}}$ \\
\hline TRAP & 557 amino acids & Whole & in from $\mathrm{T} 9$ & strain \\
\hline
\end{tabular}

Because of the many possible vaccination regimens within the prime-boost strategy, varying dose, number of priming vaccinations, interval between vaccinations, route and number of boosting vaccinations, we decided to evaluate a large number of diverse regimens in small-scale trials to try and rapidly identify a highly immunogenic regime.

\section{RESULTS}

Vaccinations

The malarial DNA sequence was full-length $P$. falciparum TRAP of strain T9/96 (ref. 30), fused to a string of 20 selected T-cell and B-cell

Table 1 Vaccination schedule of the 63 volunteers

\begin{tabular}{|c|c|c|c|c|c|c|c|c|c|c|}
\hline \multirow[t]{3}{*}{ Group } & \multirow{3}{*}{$\begin{array}{l}\text { Vaccine } \\
\text { group } \\
\text { size }\end{array}$} & \multicolumn{3}{|c|}{ DNA } & \multirow{3}{*}{$\begin{array}{l}\text { Interval } \\
\text { to } \\
\text { boost }\end{array}$} & \multicolumn{3}{|c|}{ MVA } & \multirow{3}{*}{$\begin{array}{c}\text { Interval } \\
\text { to } \\
\text { challenge }\end{array}$} & \multirow{3}{*}{$\begin{array}{c}\text { Challenge } \\
\text { group } \\
\text { size }\end{array}$} \\
\hline & & Dose & Dose & Dose & & $\times 10^{7}$ & $\times 10^{7}$ & $\times 10^{7}$ & & \\
\hline & & $(\mu \mathrm{g})$ & $(\mu \mathrm{g})$ & $(\mu \mathrm{g})$ & & PFU & PFU & PFU & & \\
\hline $\operatorname{DDD}(0.5)$ & 3 & 500 & 500 & 500 & & & & & & \\
\hline GGG & 4 & 4 & 4 & 4 & & & & & & \\
\hline M & 1 & & & & & 3 & & & & \\
\hline MMM(3) & 10 & & & & & 3 & 3 & 3 & $2-3$ & 4 \\
\hline DDDMMM(3) & 3 & 500 & 500 & 500 & $6-12$ & 3 & 3 & 3 & & \\
\hline GGGMM(3) & 2 & 4 & 4 & 4 & 14 & 3 & 3 & & & \\
\hline $\mathrm{DDD}(1)$ & 5 & 1,000 & 1,000 & 1,000 & & & & & $2-3$ & 5 \\
\hline $\mathrm{D}(1) \mathrm{MM}(3)$ & 3 & 1,000 & & & 3 & 3 & 3 & & $2-3$ & 3 \\
\hline $\mathrm{DDD}(1) \mathrm{M}(3)$ & 3 & 1,000 & 1,000 & 1,000 & 3 & 3 & & & & \\
\hline $\mathrm{DD}(1) \mathrm{MM}(3)$ & 3 & 1,000 & 1,000 & & 3 & 3 & 3 & & 5.4 & 3 \\
\hline GGMM(3) & 6 & 4 & 4 & & 3 & 3 & 3 & & 5.4 & 6 \\
\hline $\operatorname{MM}(6)$ & 3 & & & & & 6 & 6 & & & \\
\hline$M M(15)$ & 8 & & & & & 15 & 15 & & & \\
\hline DDD_MM(15) & 5 & 2,000 & 2,000 & 2,000 & 8 & 15 & 15 & & $2-3$ & 4 \\
\hline DDDMM(15) & 4 & 2,000 & 2,000 & 2,000 & 3 & 15 & 15 & & 3 & 4 \\
\hline
\end{tabular}

Repeat doses of the same vaccine were given at 3-week intervals. Intervals are measured in weeks. D, DNA.ME-TRAP given by intramuscular injection into deltoid muscle; G, DNA.ME-TRAP given epidermally by needleless delivery device; M, MVA.ME-TRAP given by intradermal injection; PFU, plaque forming unit. 
ARTICLES

Table 2 Cellular immune responses after vaccination

\begin{tabular}{|c|c|c|c|c|c|c|c|c|c|c|c|c|c|}
\hline \multirow[b]{2}{*}{$\begin{array}{l}\text { Vaccine } \\
\text { regimen }\end{array}$} & \multirow[b]{2}{*}{$\begin{array}{c}\text { Group } \\
\text { size }\end{array}$} & \multicolumn{4}{|c|}{ All peptides in vaccines } & \multicolumn{4}{|c|}{ T9/96 TRAP } & \multicolumn{4}{|c|}{ 3D7 TRAP } \\
\hline & & Mean & s.e.m. & Geomean & s.e.m. & Mean & s.e.m. & Geomean & s.e.m. & Mean & s.e.m. & Geomean & s.e.m. \\
\hline Baseline & 65 & 43 & 7 & 18 & 4 & 25 & 5 & 9 & 2 & 33 & 6 & 15 & 3 \\
\hline $\operatorname{DDD}(0.5)$ & 4 & 73 & 18 & 66 & 19 & 48 & 20 & 33 & 23 & & & & \\
\hline G & 10 & 112 & 36 & 65 & 29 & 78 & 30 & 35 & 19 & 13 & 5 & 9 & 7 \\
\hline GG & 10 & 91 & 30 & 50 & 23 & 57 & 21 & 31 & 14 & 17 & 5 & 14 & 6 \\
\hline GGG & 4 & 72 & 20 & 63 & 24 & 58 & 19 & 45 & 26 & & & & \\
\hline MMM(3) & 9 & 110 & 48 & 44 & 36 & 41 & 13 & 24 & 14 & 16 & 3 & 14 & 4 \\
\hline DDDMMM(3) & 3 & 77 & 24 & 70 & 25 & 38 & 18 & 28 & 23 & & & & \\
\hline GGGMM(3) & 2 & 92 & 78 & 50 & 120 & 15 & 8 & 13 & 9 & & & & \\
\hline$D(1)$ & 13 & 34 & 11 & 18 & 8 & 19 & 6 & 11 & 4 & 22 & 6 & 13 & 5 \\
\hline $\mathrm{DD}(1)$ & 9 & 74 & 35 & 27 & 21 & 60 & 28 & 21 & 16 & 38 & 17 & 14 & 10 \\
\hline $\mathrm{DDD}(1)$ & 8 & 55 & 23 & 33 & 16 & 44 & 21 & 25 & 12 & 55 & 23 & 28 & 17 \\
\hline $\mathrm{D}(1) \mathrm{MM}(3)$ & 3 & 112 & 68 & 69 & 78 & 55 & 24 & 43 & 29 & 25 & 13 & 16 & 18 \\
\hline $\operatorname{DDD}(1) \mathrm{M}(3)$ & 3 & 180 & 122 & 104 & 118 & 162 & 112 & 90 & 109 & 75 & 52 & 46 & 45 \\
\hline $\mathrm{DD}(1) \mathrm{MM}(3)$ & 3 & 79 & 41 & 51 & 56 & 69 & 41 & 21 & 76 & 56 & 36 & 18 & 60 \\
\hline GGM(3) & 6 & 297 & 108 & 170 & 124 & 266 & 100 & 148 & 110 & 127 & 41 & 87 & 46 \\
\hline GGMM(3) & 6 & 288 & 83 & 234 & 77 & 265 & 80 & 212 & 73 & 128 & 44 & 85 & 47 \\
\hline$M(6)$ & 2 & 109 & 85 & 68 & 126 & 44 & 27 & 35 & 36 & 208 & 129 & 163 & 174 \\
\hline $\operatorname{MM}(6)$ & 1 & 195 & & 195 & & 119 & & 119 & & 139 & & 139 & \\
\hline$M(15)$ & 2 & 5 & 5 & 3 & 7 & 3 & 3 & 2 & 4 & 12 & 5 & 11 & 6 \\
\hline MM(15) & 4 & 83 & 24 & 74 & 24 & 50 & 10 & 47 & 11 & 32 & 7 & 29 & 9 \\
\hline$D(2)$ & 7 & 22 & 2 & 21 & 2 & 13 & 3 & 10 & 5 & 25 & 5 & 21 & 7 \\
\hline $\mathrm{DDD}(2)$ & 8 & 19 & 5.7 & 14 & 5 & 12 & 4 & 8 & 3 & 33 & 19 & 14 & 9 \\
\hline DDD_M(15) & 4 & 684 & 474 & 372 & 289 & 528 & 350 & 302 & 226 & 461 & 363 & 195 & 194 \\
\hline $\operatorname{DDDM}(15)$ & 4 & 1,430 & 654 & 708 & 1,030 & 1,249 & 593 & 617 & 880 & 1,078 & 555 & 363 & 881 \\
\hline DDD_MM(15) & 5 & 188 & 53 & 158 & 53 & 150 & 29 & 137 & 34 & 118 & 35 & 98 & 36 \\
\hline DDDMM(15) & 2 & 470 & 340 & 316 & 471 & 422 & 304 & 295 & 435 & 294 & 231 & 182 & 340 \\
\hline
\end{tabular}

Data for some time points are missing because of subject unavailability, errors in performing the assay or background responses of more than 50 spots per million PBMCs, which were not included in the analysis. IFN- $\gamma$ ELISPOT responses are shown as spot-forming cells per million PBMCs, in peripheral blood $7 \mathrm{~d}$ after various vaccination regimens. The number of subjects in each arm and their vaccination schedule are shown. Some subjects are included more than once as the results indicate their time course through the trials. Arithmetic and geometric (geomean) means and standard error of the mean (s.e.m.) are shown for three sets of peptide pools: the summed net responses to all the epitopes in the vaccine insert, the summed net responses to all peptide pools from the T9/96 strain of TRAP and the summed net responses to all peptide pools from the 3D7 strain of TRAP.

epitopes $^{23}$ (ME; Fig. 1). TRAP is one of the major antigens of the preerythrocytic parasite, with a protective homolog in a rodent malaria parasite $^{18}$. The string of epitopes, but not TRAP, was recoded to a mammalian codon bias ${ }^{23}$. Healthy malaria-naive adult volunteers resident in Oxford were recruited ${ }^{31}$ and immunized with plasmid DNA and MVA vaccines recombinant for the ME-TRAP fusion protein (called DNA.ME-TRAP and MVA.ME-TRAP, respectively), individually and using heterologous prime-boost immunization regimes at a range of doses. The sequential trials were designed to explore the number of priming vaccinations and boosting vaccinations required, and to incorporate dose-ranging studies. The vaccination schedule of each arm of each trial is described in Table 1. Briefly, three groups received DNA only, four groups received MVA only at various doses and eight groups received DNA prime and MVA boost with variable intervals. The doses and number of immunizations are described in Table 1. In humans, the efficacy of pre-erythrocytic vaccines against malaria can be evaluated by sporozoite challenge with live membrane-fed infectious mosquitoes. Of the vaccinated subjects, seven groups had sporozoite challenge as described below. DNA.ME-TRAP was given either intramuscularly at doses of $500,1,000$ or $2,000 \mu \mathrm{g}$ or epidermally by a needleless delivery device (Powderject) at a dose of $4 \mu \mathrm{g}$ (ref.
15). MVA.ME-TRAP was given by intradermal injections of $100 \mu \mathrm{l}$ aliquots into the skin over one or both deltoid areas at doses of 3, 6 or $15 \times 10^{7}$ plaque-forming units (PFU). The number in brackets in the group name corresponds to the dose of vaccine. For example, $\operatorname{GGMM(3)}$ indicates that the DNA vaccine was administered twice by needleless delivery device followed twice by $3 \times 10^{7}$ PFU of MVA.METRAP. Table 1 lists and clarifies the other abbreviations used to indicate vaccinations. The vaccines were well tolerated ${ }^{31}$. Intramuscular DNA vaccination was not associated with any localized adverse events. No antinuclear antibodies were detected after vaccination.

\section{Cellular responses are higher after prime-boost vaccination}

Repeated vaccination with DNA alone produced small responses in the ex vivo ELISPOT assay $(P=0.06)$, but addition of a subsequent booster immunization with MVA.ME-TRAP led to a massive increase in the responses (Table 2 and Fig. 2). After vaccination, the summed net spots in ELISPOT wells to peptides from P. falciparum T9/96strain TRAP, in subjects who had a DNA prime followed by MVA.METRAP, showed a significant change from baseline $(P=0.0006$, with adjustment for multiple comparisons). The cross-strain responses to pools of peptides from $P$. falciparum 3D7-strain TRAP were lower than 


\section{ARTICLES}

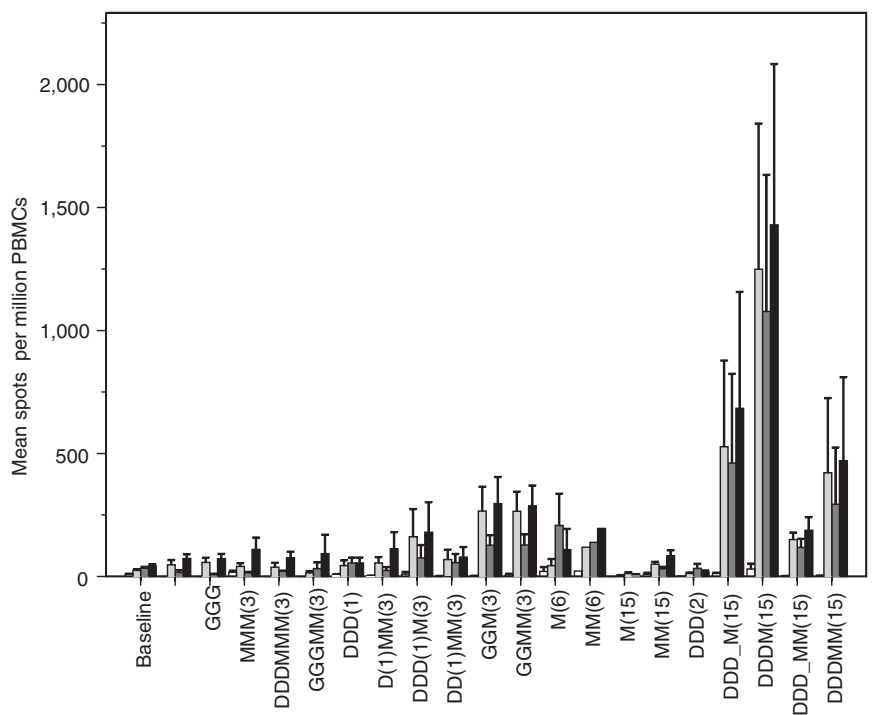

the responses to pools of peptides from the $P$. falciparum T9/96 vaccine strain, but still changed from baseline. Low responses were found to the octamer, nonamer and decamer peptides in the ME string with significant responses only in the groups that received three doses of $2 \mathrm{mg}$ DNA and a subsequent MVA boost at a dose of $15 \times 10^{7} \mathrm{PFU}$.

\section{Dosage and timing of vaccination affect immunogenicity}

The doses of DNA and MVA are important; higher doses were associated with much higher responses. Also, prime-boost immunization using epidermal delivery of $4 \mu \mathrm{g}$ DNA by needleless device followed by
Figure 2 ELISPOT responses to pools of peptides $7 \mathrm{~d}$ after various vaccination regimens. Shown are summed net responses to pools of peptides comprising the $14 \mathrm{HLA}$ class I epitopes in the polyepitope string $(\square)$, the T9/96 strain of TRAP $(\square)$, the 3D7 strain of TRAP $(\square)$ and the entire insert

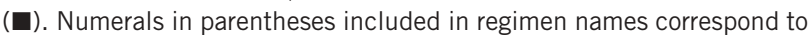
dosage of vaccine, as indicated in Table 1. Error bars represent s.e.m.. In many cases, a single subject is included in several regimens in this figure. For example, a subject who had three doses of $2 \mathrm{mg}$ of DNA.ME-TRAP followed 3 weeks later by two doses of $15 \times 10^{7}$ PFU of MVA.ME-TRAP is included in the $\mathrm{DDD}(2)$ regimen $7 \mathrm{~d}$ after the first three vaccinations, in the $\operatorname{DDDM}(15)$ regimen $7 \mathrm{~d}$ after the first MVA.ME-TRAP and in the DDDMM(15) regimen $7 \mathrm{~d}$ after the second MVA.ME-TRAP.

a low dose of MVA may be more immunogenic than intramuscular delivery of $1 \mathrm{mg}$ DNA followed by the same MVA dose $(P=0.10)$. When using the higher doses of DNA and MVA, we did not find evidence that the longer interval of 8 weeks between DNA and MVA was any better than a 3 -week interval. The immunological responses after the shorter interval of 3 weeks between DNA and MVA were higher $(P$ $=0.026$ ) than after an 8-week interval.

\section{Immune responses persist for several months}

The T-cell responses waned over time, but in the GGMM(3) group they were still $36 \%$ of the peak after $5-11$ months and $53 \%$ of the initial plateau level (days 21-28) at the 5- to 11-month time point (Fig. 3a). In the DDDMM(15) and DDD_MM(15) groups, 156 T9/96 TRAP-specific spots per million peripheral blood mononuclear cells (PBMCs) were found after 6 months (69\% of the day 7 level).

\section{Prime-boost vaccination shows some efficacy}

The P. falciparum sporozoite challenge model we adopted ${ }^{32}$ used gametocyte culture and membrane feeding ${ }^{33}$. For the first time in subunit vaccine studies, we used a different strain of parasite for challenge a

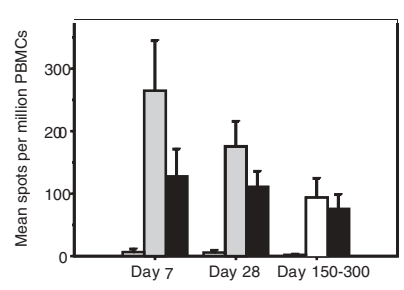

b

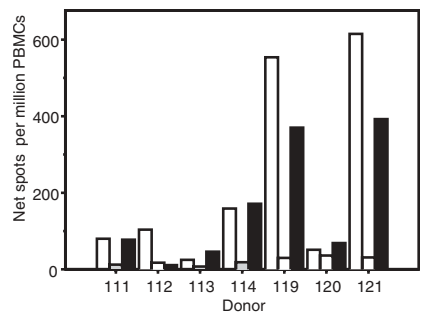

C

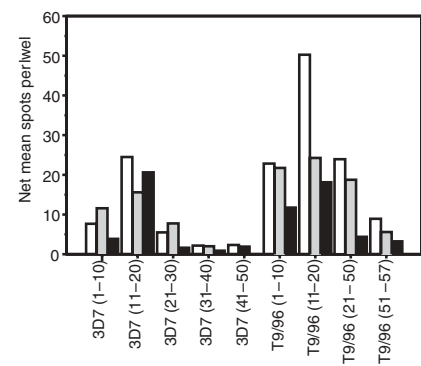

d

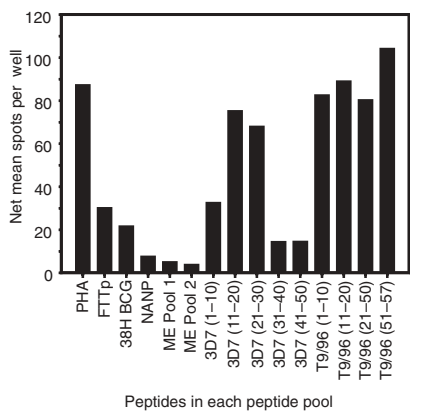

Figure 3 Characteristics of induced T-cell responses and protective efficacy. (a) Time course at 7, 28 and 150-350 d after vaccination for six subjects in group GGMM(3). $\square$, ME epitopes; $\square$, TRAP T9/96 peptides; $\square$ TRAP 3D7 peptides. The arithmetic mean and standard error are shown. (b) The results of CD4 ${ }^{+}$and $C D 8^{+} T_{-}^{-}$ cell depletion experiments from DDD_MM(15) and DDDMM(15) groups $7 \mathrm{~d}$ after the last vaccine. $\square$, undepleted; $\square$, CD4-depleted; $\mathbf{\square}$, CD8-depleted. (c,d) Breadth of the induced responses. The responses to each peptide pool shown in $\mathbf{c}$ are from the same subjects and time points as in $\mathbf{a} . \square$, day 7 ; $\square$, day 28; $\mathbf{\square}$, days 150-300. Data in $\mathbf{d}$ are $7 \mathrm{~d}$ after the first MVA booster dose of $15 \times 10^{7} \mathrm{PFU}$, for those who had three priming doses of $2 \mathrm{mg}$ of DNA.ME-TRAP intramuscularly. The nomenclature of the peptide pools is described in Fig. $\mathbf{1 b}$ and in the footnote to Table 3. (e) Kaplan-Meier curves of time from sporozoite challenge to parasitemia detected on thick blood film for three groups: 16 unvaccinated control subjects, 14 heterologous prime-boost vaccinated subjects who received either $\operatorname{GGMM}(3)(n=6)$, DDDMM(15) $(n=4)$ or DDD_MM(15) $(n=4$; these being the groups with the strongest immune responses), and 9 vaccinated subjects who received just one of the vaccines, either MMM(3) ( $n=4)$ or $\mathrm{DDD}(1)(n=5$; Table 1$)$. HP-B, heterologous prime-boost. Development of parasitemia is delayed significantly in the heterologous prime-boost immunization group (log rank test, $P=$ 0.013 ) compared with the unvaccinated controls, but there is no significant difference in time to parasitemia when comparing the volunteers who received DNA only or MVA only and the unvaccinated group. e

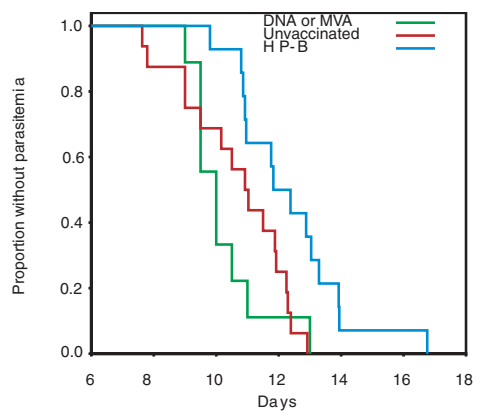


Table 3 Correlation between time to parasitemia and immune responses to various regions of the vaccine antigens

\begin{tabular}{lcc}
\hline Peptide pool & Pearson's correlation coefficient & $P$ value \\
\hline PHA & 0.100 & 0.538 \\
FTTp & 0.130 & 0.421 \\
BCG & 0.256 & 0.110 \\
NANP & -0.053 & 0.745 \\
ME1 & 0.327 & 0.039 \\
ME2 & 0.027 & 0.865 \\
3D7(1-10) & 0.202 & 0.211 \\
3D7(11-20) & 0.180 & 0.265 \\
3D7(21-30) & 0.240 & 0.136 \\
3D7(31-40) & 0.420 & 0.007 \\
3D7(41-50) & 0.165 & 0.306 \\
T9/96(1-10) & 0.280 & 0.080 \\
T9/96(11-20) & 0.212 & 0.187 \\
T9/96(21-50) & 0.365 & 0.020 \\
T9/96(51-57) & 0.235 & 0.143 \\
\hline
\end{tabular}

Transformation of the time to parasitemia to a logarithmic scale did not change the results. PHA, phytohemagglutinin; FTTp and BCG NANP, as in Fig. 1 and ref 23; ME1, pool of first 7 epitopes in the multiple epitope string; ME2, pool of the other 7 epitopes; 3D7 $(x-y)$ refers to a pool of ten overlapping 20-mer peptides spanning 110 amino acids of the 3D7 strain of TRAP ending at the $(y \times 10)+10$ amino acids, and T9/96( $x-y)$ refers to a pool of overlapping 20-mer peptides spanning a corresponding region of the T9/96 vaccine strain of TRAP.

(P. falciparum 3D7 strain) from that of the vaccine antigen (TRAP of the T9/96 strain). Volunteers in the GGMM(3), DDD_MM(15) (8week interval between third DNA and first MVA) and DDDMM(15) (3-week interval) groups, but not those receiving homologous boost immunization regimes, had a significant delay in time to parasitemia $(P=0.013$; Fig. 3e). Calculation of the likely reduction in hepatic parasite load required to effect a $2-\mathrm{d}$ mean delay in time to microscopically detectable blood stage parasitemia suggests a $>70 \%$ reduction in liver-stage parasites in these vaccinees. Based on an approximately eightfold multiplication rate of blood-stage parasites in one 48-h cycle in human blood ${ }^{34}$, it may be estimated that a 2 -d delay in time to detectable parasitemia corresponds to an approximately $87.5 \%$ (100 $100 / 8=87.5)$ reduction in the number of viable parasites emerging from the liver ${ }^{35}$. The reduction in sporozoite dose required to effect a 48-h delay in time to detectable blood stage parasitemia in the murine Plasmodium berghei model is 75\% (R.J. Anderson and A.V.S.H., unpublished data). Thus, these vaccination regimes induced an effective immune response against pre-erythrocytic $P$. falciparum parasites.

\section{Both $\mathrm{CD}^{+}{ }^{+}$and $\mathrm{CD} 8^{+}$T-cell responses after vaccination}

As anticipated from preclinical studies of DNA-MVA immunization, both $\mathrm{CD}^{+}$(refs. 18,25,36) and $\mathrm{CD}^{+}$(ref. 20) IFN- $\gamma$-producing cells were induced, in contrast to use of a previous plasmid DNA vaccine alone that primarily induced $\mathrm{CD} 8^{+} \mathrm{T}$ cells ${ }^{13,14}$. In the GGMM(3) group, depletion assays on cryopreserved cells showed that four of the subjects had $\mathrm{CD}^{+}{ }^{+} \mathrm{T}$-lymphocyte-dependent responses and two had $\mathrm{CD} 8^{+} \mathrm{T}$ lymphocyte-dependent responses. In the $\operatorname{DDDMM}(15)$ and DDD_MM(15) groups, depletion of fresh cells on samples taken $7 \mathrm{~d}$ after vaccination showed that the responses were mainly $\mathrm{CD} 4^{+} \mathrm{T}-\mathrm{lym}-$ phocyte-dependent (Fig. 3b). At the prevaccination tests, all but one of the peptide pools had mean values less than five spots per million PBMCs. However, the mean net spots for the TRAP 3D7 peptide pool of peptides 21-30 had 52 spots per million PBMCs. As none of the volun- teers had had malaria exposure, this suggests the presence of some crossreactive epitopes in this pool. The responses were broad, with responses detected to all peptide pools tested (Fig. 3c,d). Responses of some peptide pools, such as pool 31-40 from TRAP (corresponding to amino acids 300-410), showed a significant correlation with time to parasitemia without correcting for multiple comparisons (Table 3). Thus, these responses may contribute to the protective effect of the vaccine.

Induced antibody responses were very limited. One vaccinated subject had a fourfold rise in the titer of antibody to TRAP, and two others had a twofold rise in titer. Four subjects developed low-titer antibodies to the NANP repeat epitope in the vaccine. These low-titer antibody responses did not correlate with protection.

\section{DISCUSSION}

This is the first demonstration of protective antimalarial T-cell responses in humans, induced by vaccination in the absence of significant antibodies. The frequency of circulating effector T cells, as measured by ex vivo ELISPOT, was much higher than in other vaccination studies in humans. For example, after RTS,S/AS02 malaria vaccination, the comparable geometric mean response in the most responsive subgroup was about 20 cells per million PBMCs, compared with a geometric mean for the DDDM group of 708 (ref. 17). A DNA vaccine for HBV elicited protective levels of antibodies and some cellular responses, but use of a different methodology to measure cellular immune responses limits direct comparison ${ }^{15}$. Another malaria DNA vaccine $^{14}$ shows similar immunogenicity in ex vivo ELISPOT to DNA.ME-TRAP, but the response is 7- or 15-fold lower than DNA/MVA prime-boost immunization with DDD_M(15) or $\operatorname{DDDM}(15)$, respectively. An earlier study of cellular immunity required prestimulation of lymphocytes to elicit detectable responses ${ }^{13}$. The results indicate that DNA priming followed by MVAboosting vaccination produces $\mathrm{T}$-cell responses in humans that far surpass in magnitude responses seen after either vaccine alone.

Partial protection manifesting as delayed parasitemia was achieved even though challenge used a different strain of $P$. falciparum, a higher dose of sporozoites and a larger number of infectious bites than might be expected in the field. The TRAP amino acid sequences from T9/96 and 3D7 strains of P. falciparum show $6.1 \%$ sequence difference, more than is typically found between pairs of isolates from Africa ${ }^{37}$. A mosquito bite was not scored as positive unless more than 100 sporozoites were observed in each dissected salivary gland. Quantitative PCR analysis of blood-stage parasites emerging from the liver suggests that such a challenge regime may administer about ten times more sporozoites than a natural field mosquito bite ${ }^{38,39}$. A vaccine aimed at protecting against pre-erythrocytic stages requires sterile immunity. The delay in parasitemia observed probably reflects elimination of a large proportion of the sporozoite-infected hepatocytes, and this capacity might be adequate to provide sterile immunity in a field setting where fewer sporozoites may commonly be inoculated. This was the first time that two different strains of $P$. falciparum (one for vaccination and one for challenge) were used in a subunit malaria vaccine trial, and the protection seen in this study may translate to greater field efficacy than data from homologous-strain malaria challenge studies. However, the level of protection observed here is less than was observed after challenge of RTS,S vaccinees with the same parasite strain as that used in the candidate vaccine (homologous strain challenge) ${ }^{40}$, perhaps because RTS,S induces high-level antibody responses.

These are the first vaccines expressing a complex polyepitope string to be evaluated in humans, and responses were seen to multiple epitopes in the string, indicating that these epitopes are successfully processed and presented in humans. Stronger responses, 


\section{ARTICLES}

however, were seen to the 557-amino-acid TRAP protein than to the 232-amino-acid polyepitope string, suggesting that TRAP contains either more abundant or more dominant epitopes in this construct.

We have shown that vaccination using the TRAP sequence from the T9/96 strain of $P$. falciparum generates peptide-specific T cells that respond to TRAP peptides from the alternative 3D7 $P$. falciparum strain used in the challenge. Better crossreactivity was observed with the higher dose and more immunogenic regimes, for example $\operatorname{GGMM}(3)$ and $\operatorname{DDDM}(15)$ (Fig. 2). This indicates a probable immunological basis for the observed cross-protection against the different strain 3D7 P. falciparum. The persistence of the responses was also impressive, as the levels $150-350 \mathrm{~d}$ after the last vaccination were $61 \%$ of the day 21-28 levels. Measurement by the same ELISPOT method of T-cell responses to TRAP present in adults in malariaendemic areas of East and West Africa has shown a mean level of 10-30 spots per million PBMCs ${ }^{34,35,41}$. Thus, the DNA-MVA vaccineinduced responses are substantially greater than those generated by decades of natural exposure to malaria.

Preclinical data suggest that recombinant MVA is a particularly effective agent for boosting T-cell responses ${ }^{18,20,42}$. We show that in humans, a single dose of recombinant MVA is adequate and little further benefit seems to be gained from subsequent booster immunizations. MVA is an increasingly promising viral vector due to its marked immunogenicity when used as a boosting agent, its excellent safety profile in an immunocompromised macaque study ${ }^{43}$ and its safety in humans ${ }^{31}$.

Although initial studies in animals showed considerable promise for DNA vaccination, clinical trial results have been generally disappointing. In contrast, the heterologous prime-boost approach used here shows much greater T-cell immunogenicity in humans and could offer a basis for effective T-cell induction against many infectious pathogens as well as some malignancies.

\section{METHODS}

The DNA.ME-TRAP vaccine. Within the plasmid conferring kanamycin resistance, the ME-TRAP hybrid was regulated by a cytomegalovirus immediateearly promoter with intron A for expression in eukaryotic cells and a bovine growth hormone-derived polyadenylation transcription terminator. DNA.ME-TRAP was produced under good manufacturing practices by Qiagen $\mathrm{GmbH}$. FTTp is the universal helper epitope from tetanus toxoid protein, with a phenylalanine substituted for the tyrosine.

The MVA.ME-TRAP vaccine. The ME-TRAP hybrid DNA was ligated into the vaccinia shuttle vector pSC11, bringing it under the control of the vaccinia P7.5 early/late promoter. This vector included the Escherichia coli $\beta$-galactosidase gene expressed by the vaccinia P11 late promoter. The region, including METRAP and the $\beta$-galactosidase gene, is flanked by sequences from the vaccinia thymidine kinase locus to allow insertion into the vaccinia genome. Chicken embryo fibroblast cells infected with wild-type MVA virus were transfected with pSC11 ME-TRAP. Recombinant virus was isolated using $\beta$-galactosidase substrate X-gal (5-bromo-4-chloro-3-indolyl-D-galactopyranoside) overlay of infected chicken embryo fibroblast monolayers ${ }^{44}$. Clinical grade MVA.METRAP was produced under good manufacturing practices by Impfstoffwerke Dessau-Tornau.

Clinical trials. Volunteers were recruited for both immunization and challenge studies under protocols approved by the Oxfordshire Research Ethics Committee and enrolled only after obtaining written informed consent. The sequence of vaccinations was carried out by moving from lower to higher dose and from testing each agent individually to testing sequential combinations of vaccines (Table 1).

Immunogenicity measures. The main immunological measure used to determine vaccine immunogenicity was the ex vivo IFN- $\gamma$ ELISPOT response, which correlates with protection in mouse sporozoite challenge studies. This was per- formed at baseline, 7, 21-28 and 150-300 d after vaccination. These measurements were carried out on fresh PBMCs using pools of 20-mer peptides that span the length of TRAP and overlap by 10 amino acids (W.H.R. and J.M.V., unpublished data). The known epitopes in the ME string (Fig. 1b and ref. 23) were also tested in pools. Briefly, 400,000 PBMCs per well were plated directly onto the ELISPOT plate (MAIP, Millipore) in the presence of $25 \mu \mathrm{g} / \mathrm{ml}$ of each peptide and incubated for $18 \mathrm{~h}$. ELISPOT responses to TRAP peptides of the vaccine strain, T9/96, and to the challenge strain, 3D7, were assessed separately. The 57 T9/96 TRAP peptides were tested in four pools and the 3D7 TRAP peptides were tested in six pools (Fig. 3c). The promiscuous HLA class II-binding peptides from bacillus Calmette-Guérin and tetanus toxoid were tested separately. Assays were performed in duplicate and the results were averaged. Antibodies to the CSP NANP repeat sequence and to TRAP of both the 3D7 and T9/96 strains were measured by ELISA.

Analysis of immunogenicity. ELISPOT assays in which more than 50 spots per million PBMCs were present with medium and cells alone were not included in the analysis. The ELISPOT data were analyzed by subtracting the number of spots in the wells with medium and cells alone from the counts of spots in wells with pools of peptides and cells. Counts less than zero were disregarded. The results were summed across all the peptide pools for one donor at one time point. This will count twice a $\mathrm{T}$ cell that responds to any of the 10-mer overlap regions that occur in two pools with adjacent peptides. The sensitivity of the analysis was maximized by summing across all peptide pools after wells with negative values were fixed to zero, although the absolute number of reactive cells may have been slightly inflated. Arithmetic and geometric means of the summed peptide-specific spots are presented with the standard error of the mean. Analysis of variance for repeated measurements was used to compare between groups and to compare postvaccination with baseline measures. This model uses the cellular immune response for several time points for each subject to maximize the power to detect an effect. At the same time, it models the dependency within an individual's repeated measures.

Challenge. Five Anopheles stephensi mosquitoes, each with $10^{2}-10^{4}$ sporozoites per salivary gland, were allowed to bite each subject, thus delivering 3D7 strain P. falciparum sporozoites ${ }^{33}$. Challenges took place $14-37 \mathrm{~d}$ after the final vaccination. Monitoring took place twice daily using Giemsa-stained thick blood films starting on day 5 . Subjects were treated with chloroquine after the first confirmed positive blood film. The five or six unvaccinated control subjects in each challenge trial all developed parasitemia 8-13 d later. Data were pooled from three similarly performed challenge studies at different time points, each with 5-6 unvaccinated controls and 9-12 vaccinees. There was no significant difference in time to parasitemia between the control groups in these three challenges. The time to parasitemia between groups of subjects was compared using the log rank test. No significant differences were observed between the pooled unvaccinated controls and the $\mathrm{DDD}(1), \mathrm{D}(1) \mathrm{MM}(3), \mathrm{DD}(1) \mathrm{MM}(3)$ and $\operatorname{MMM}(3)$ groups (Table 1), all of which showed modest immunogenicity (Fig. 2 and Table 2). In contrast, the heterologous prime-boost groups GGMM(3), DDDMM(15) and DDD_MM(15), which showed high immunogenicity ( $>150$ SFU $/ 10^{6}$ PBMCs; Fig. 2 and Table 2 ), showed a significant delay in time to parasitemia (Fig. 3e).

\section{ACKNOWLEDGMENTS}

We acknowledge the contribution made to this research by the subjects who volunteered to participate in this study. We thank M. Plebanski, A. J. McMichael, R. J. Anderson, P. Degano, M. Pinder, G. Cooke, D. Crook, A. Neubert and S. Correa for discussions and assistance, and the Wellcome Trust for funding the work. S.J.M. and V.S.M. are Wellcome Trust Tropical Medicine Fellows, and G.L.S. and A.V.S.H. are Wellcome Trust Principal Research Fellows.

\section{COMPETING INTERESTS STATEMENT}

The authors declare competing financial interests (see the Nature Medicine website for details).

Received 7 January; accepted 25 April 2003

Published online 25 May 2003; doi:10.1038/nm881

1. Harty, J.T., Tvinnereim, A.R. \& White, D.W. CD8 ${ }^{+}$T cell effector mechanisms in resistance to infection. Annu. Rev. Immunol. 18, 275-308 (2000). 
2. Kaech, S.M., Wherry, E.J. \& Ahmed, R. Effector and memory T-cell differentiation: implications for vaccine development. Nat. Rev. Immunol. 2, 251-262 (2002).

3. Parker, D.C. T cell-dependent B cell activation. Annu. Rev. Immunol. 11, 331-360 (1993).

4. Doolan, D.L. \& Hoffman S.L. The complexity of protective immunity against liver-stage malaria. J. Immunol. 165, 1453-1462 (2000).

5. Guidotti, L.G. et al. Intracellular inactivation of the hepatitis B virus by cytotoxic T lymphocytes. Immunity 4, 25-36 (1996).

6. Villinger, F. et al. Adoptive transfer of simian immunodeficiency virus (SIV) naive autologous $\mathrm{CD}^{+}$cells to macaques chronically infected with SIV is sufficient to induce long-term nonprogressor status. Blood 99, 590-599 (2002).

7. Winter, H., Hu, H.M., Urba, W.J. \& Fox, B.A. Tumor regression after adoptive transfer of effector $T$ cells is independent of perforin or Fas ligand (APO-1L/CD95L). J. Immunol. 163, 4462-4472 (1999).

8. Becker, C. et al. Adoptive tumor therapy with T lymphocytes enriched through an IFN$\gamma$ capture assay. Nat. Med. 7, 1159-1162 (2001)

9. Seki, N. et al. Tumor-specific CTL kill murine renal cancer cells using both perforin and Fas ligand-mediated lysis in vitro, but cause tumor regression in vivo in the absence of perforin. J. Immunol. 168, 3484-3492 (2002).

10. Zwaveling, S. et al. Established human papillomavirus type 16-expressing tumors are effectively eradicated following vaccination with long peptides. J. Immunol. 169, 350-358 (2002)

11. Bertoletti, A. et al. HLA class I restricted human cytotoxic T cells recognise endogenously synthesised hepatitis B virus nucleocapsid antigen. Proc. Natl. Acad. Sci. USA 88, 10445-10449 (1991).

12. Boni, C. et al. Lamivudine treatment can restore T cell responsiveness in chronic hepatitis B. J. Clin. Invest. 102, 968-975 (1998)

13. Wang, R. et al. Induction of antigen-specific cytotoxic T lymphocytes in humans by a malaria DNA vaccine. Science 282, 476-480 (1998).

14. Wang, R. et al. Induction of CD4+ T cell-dependent $C D 8^{+}$type 1 responses in humans by a malaria DNA vaccine. Proc. Natl. Acad. Sci. USA 98, 10817-10822 (2001).

15. Roy, M.J. et al. Induction of antigen-specific $C D 8^{+} \mathrm{T}$ cells, $\mathrm{T}$ helper cells, and protective levels of antibody in humans by particle-mediated administration of a hepatitis $B$ virus DNA vaccine. Vaccine 19, 764-778 (2000).

16. Gupta, K. et al. Safety and immunogenicity of a high-titered canarypox vaccine in combination with rgp120 in a diverse population of HIV-1-uninfected adults: AIDS Vaccine Evaluation Group Protocol 022A. J. Acquir. Immune Defic. Syndr. 29, 254-261 (2002)

17. Lalvani, A. et al. Potent induction of focused Th1-type cellular and humoral immune responses by RTS/S/SBAS2, a recombinant Plasmodium falciparum malaria vaccine. J. Infect. Dis. 180, 1656-1664 (1999).

18. Schneider, J. et al. Enhanced immunogenicity for $\mathrm{CD}^{+} \mathrm{T}$ cell induction and complete protective efficacy of malaria DNA vaccination by boosting with modified vaccinia virus Ankara. Nat. Med. 4, 397-402 (1998).

19. Hanke, T. et al. Enhancement of MHC class I-restricted peptide-specific T cell induction by a DNA prime/MVA boost vaccination regime. Vaccine 16, 439-445 (1998).

20. McShane, H., Brookes, R., Gilbert, S.C. \& Hill, A.V. Enhanced immunogenicity of $\mathrm{CD}^{+}$T-cell responses and protective efficacy of a DNA-modified vaccinia virus Ankara prime-boost vaccination regimen for murine tuberculosis. Infect. Immun. 69, 681-686 (2001).

21. Schneider, J. et al. Induction of $\mathrm{CD}^{+} \mathrm{T}$ cells using heterologous prime-boost immunisation strategies. Immunol. Rev. 170, 29-38 (1999).

22. Gilbert, S.C. et al. Enhanced CD8 T cell immunogenicity and protective efficacy in a mouse malaria model using a recombinant adenoviral vaccine in heterologous primeboost immunisation regimes. Vaccine 20, 1039-1045 (2002).

23. Gilbert, S.C. et al. A protein particle vaccine containing multiple malaria epitopes. Nat. Biotechnol. 15, 1280-1284 (1997).
24. Sullivan, N.J., Sanchez, A., Rollin, P.E., Yang, Z.Y. \& Nabel, G.J. Development of a preventive vaccine for Ebola virus infection in primates. Nature 408, 605-609 (2000)

25. Amara, R.R. et al. Control of a mucosal challenge and prevention of AIDS by a multiprotein DNA/MVA vaccine. Science 292, 69-74 (2001).

26. Marshall, E. Renewed assault on an old and deadly foe. Science $\mathbf{2 9 0 , 4 2 8 - 4 3 0}$ (2000).

27. Hill, A.V.S. et al. Common West African HLA antigens are associated with protection from severe malaria. Nature 352, 595-600 (1991).

28. Herrington, D. et al. Successful immunization of humans with irradiated malaria sporozoites: humoral and cellular responses of the protected individuals. Am. J. Trop. Med. Hyg. 45, 539-547 (1991).

29. Hoffman, S.L. et al. Sporozoite vaccine induces genetically restricted T cell elimination of malaria from hepatocytes. Science 244, 1078-1081 (1989).

30. Robson, K.J. et al. A highly conserved amino-acid sequence in thrombospondin, properdin and in proteins from sporozoites and blood stages of a human malaria parasite. Nature 335, 79-82 (1988).

31. Moorthy, V.S. et al. Safety of DNA and modified vaccinia virus Ankara vaccines against liver-stage $P$. falciparum malaria in non-immune volunteers. Vaccine 21, 2004-2011 (2003).

32. Chulay, J.D. et al. Malaria transmitted to humans by mosquitoes infected from cultured Plasmodium falciparum. Am. J. Trop. Med. Hyg. 35, 66-68 (1986).

33. Ponnudurai, T., Meuwissen, J.H.E.T., Leeuwenberg, A.D.E.M., Verhave, J.P. \& Lensen, A.H.W. The production of mature gametocytes of Plasmodium falciparum in continuous cultures of different isolates infective to mosquitoes. Trans. R. Soc. Trop. Med. Hyg. 76, 242-250 (1982).

34. Simpson, J.A., Aarons, L., Collins, W.E., Jeffery, G.M. \& White, N.J. Population dynamics of untreated Plasmodium falciparum malaria within the adult human host during the expansion phase of the infection. Parasitology 124, 247-263 (2002).

35. Davis J.R. et al. Estimate of anti-Plasmodium falciparum sporozoite activity in humans vaccinated with synthetic circumsporozoite protein (NANP)3. Trans. $R$. Soc. Trop. Med. Hyg. 83, 748-750 (1989).

36. Schneider, J. et al. A prime-boost immunisation regimen using DNA followed by recombinant modified vaccinia virus Ankara induces strong cellular immune responses against the Plasmodium falciparum TRAP antigen in chimpanzees. Vaccine 19, 4595-4602 (2001).

37. Robson, K.J. et al. Natural polymorphism in the thrombospondin-related adhesive protein of Plasmodium falciparum. Am. J. Trop. Med. Hyg. 58, 81-89 (1998).

38. Hermsen, C.C. et al. Detection of Plasmodium falciparum malaria parasites in vivo by real-time quantitative PCR. Mol. Biochem. Parasitol. 118, 247-251 (2001).

39. Davis J.R. et al. Estimate of Plasmodium falciparum sporozoite content of Anopheles stephensi used to challenge human volunteers. Am. J. Trop. Med. Hyg. 40, 128-130 (1989)

40. Stoute, J.A. et al. Long-term efficacy and immune responses following immunization with the RTS, S malaria vaccine. J. Infect. Dis. 178, 1139-1144 (1998).

41. Flanagan, K. et al. Ex-vivo interferon $\gamma$ immune response to TRAP in coastal Kenyans: longevity and risk of P. falciparum infection. Am. J. Trop. Med. Hyg (in press).

42. Plebanski, M. et al. Protection from Plasmodium berghei infection by priming and boosting $T$ cells to a single class I-restricted epitope with recombinant carriers suitable for human use. Eur. J. Immunol. 28, 4345-4355 (1998).

43. Stittelaar, K.J. et al. Safety of modified vaccinia virus Ankara (MVA) in immunesuppressed macaques. Vaccine 19, 3700-3709 (2001).

44. Chakrabarti, S., Brechling, K. \& Moss, B. Vaccinia virus expression vector: coexpression of $\beta$-galactosidase provides visual screening of recombinant virus plaques. Mol. Cell. Biol. 5, 3403-3409 (1985). 